Circumplex Measures of Interpersonal Constructs

Kenneth D. Locke

\author{
Send Correspondence To: \\ Kenneth D. Locke, Ph.D. \\ Department of Psychology \\ University of Idaho \\ Moscow, ID 83844-3043 \\ (208) 885-4453 \\ (208) 885-7710 FAX \\ klocke@uidaho.edu
}

This is a pre-publication draft of a chapter to be published in the Handbook of Interpersonal Psychology (expected publication date: November, 2010) 


\section{Circumplex Measures of Interpersonal Constructs}

Kenneth D. Locke

The interpersonal circle or interpersonal circumplex has in recent decades become the most popular model for conceptualizing, organizing, and assessing interpersonal dispositions (Wiggins, 2003). The interpersonal circumplex is defined graphically by two orthogonal axes: a vertical axis (of status, dominance, power, control, or, most broadly, agency) and a horizontal axis (of solidarity, friendliness, warmth, love, or, most broadly, communion). Thus, each point within the interpersonal circumplex (IPC) can be specified as a weighted mixture of agency and communion. Simple interpersonal characteristics (such as "introverted" or "forceful") may be located graphically as a distinct combination of the two broad underlying factors; in other words, there is a particular location within the IPC space for each interpersonal disposition.

IPC inventories are inventories designed to measure interpersonal dispositions from every segment of the IPC. IPC inventories comprise a family of related instruments: All members of the family are based on the same theoretical model, but each member focuses on a different type of construct (e.g., traits, motives, problems). In this chapter, I first will summarize the IPC model that unites the diverse IPC inventories. Second, I will describe the IPC inventories that are currently in use and provide examples of how each one is being used to advance contemporary interpersonal research. Third, I will describe some simple methods for scoring, graphing, and interpreting IPC inventories, and for using IPC inventories to identify maladaptive interpersonal patterns.

\section{The Interpersonal Circumplex Model}

Multiple literatures support the centrality of agency and communion. Evolutionary psychology highlights how, throughout our evolutionary history, natural selection has favored those who could master the challenges of negotiating and coordinating both communion (e.g., attachments and coalitions) and agency (e.g., hierarchical power) (Bugental, 2000). Evidence 
that different hormones and neurotransmitters are associated with regulating communion (e.g., oxytocin; Bartz \& Hollander, 2006) and agency (e.g., testosterone; Archer, 2006) supports the view that they are both essential yet distinct tasks. From a psychometric perspective, factor analyses show that the dimensions of agency and communion account for a large proportion of the variance in ratings of interpersonal behaviors and traits (Foa, 1961; Wiggins, 1979). Additional psychometric support comes from studies showing that extraversion and agreeableness (the interpersonal factors of the solidly supported five-factor model of personality) are rotational variants of agency and communion (McCrae \& Costa, 1989).

The IPC can be divided into broad segments (such as fourths) or narrow segments (such as sixteenths), but most IPC inventories partition the circle into eight octants as shown in Figure 1. As one moves around the circle, each octant reflects a progressive blend of the two axial dimensions. By convention, each octant has a generic two-letter code (shown in parentheses in Figure 1).

To be considered an IPC inventory, an inventory's octant scales should have the following properties: (a) scales that are closer to one another on the circle should have higher correlations than scales that are farther apart; (b) the scales' communalities on the two underlying dimensions of agency and communion should all be high and approximately equal; and (c) plotting the octant scales on the two underlying axes should show them to be distributed at approximately equal 45-degree intervals. Unless otherwise noted, all of the inventories reviewed in this chapter meet these criteria (as well as the usual psychometric criteria for scale reliability and convergent validity with related measures).

For simplicity some researchers (e.g., Moskowitz, 2009) assess just the dimensions of agency and communion rather than the 8 or 16 segments assessed by the IPC inventories reviewed below. Indeed, theoretically the IPC can be defined by any two orthogonal bipolar axes that align largely within the IPC plane. Thus, to the extent that measures of the extraversion and agreeableness dimensions of Five-Factor Model can be mapped onto the 
IPC (McCrae \& Costa, 1989), such measures also can be used to locate interpersonal dispositions within the IPC space.

As described in the following section, there now exist IPC measures for many different constructs, such as traits, problems, and self-efficacy. For each inventory reviewed below, I will first provide a brief description of the inventory (e.g., purpose, length, items) and then summarize at least one example of recent research which successfully employed that inventory.

\section{Circumplex Measures of Interpersonal Constructs}

Although the IPC inventories reviewed below typically are used as self-report measures of global dispositions, they can be-and most have been-used in other ways. For example, many of the self-report measures have (with minor changes to the instructions or items) been used to obtain ratings by peer or observers. Likewise, many of the inventories have been used to assess, not only general dispositions, but also dispositions in specific situations (e.g., at work), in specific relationships (e.g., with your therapist), or under specific conditions (e.g., when under stress). Finally, short-forms are available for most of the inventories.

The first IPC measure was the Interpersonal Check List (ICL; LaForge \& Suczek, 1955). The 128-item ICL assesses 16 segments of the IPC; eight adjectives or verb-phrases assess each segment. Each item is weighted according to one of four levels of extremity. Although the ICL has been used in numerous studies (Clark \& Taulbee, 1981), it scales provide uneven coverage of the IPC space. Since the introduction of the ICL, interpersonal scales have been constructed for a variety of domains, as described below.

\section{Assessing Interpersonal Traits}

The psychometric and circumplex properties of the Interpersonal Adjective Scales (IAS; Wiggins, 1995; Wiggins, Trapnell, \& Phillips, 1988) are superior to that of the ICL and so it is now the preferred measure of interpersonal traits. IAS respondents rate each of 64 interpersonal adjectives with respect to how accurately it describes the target (usually the self) 
on 1 (very inaccurate) to 8 (very accurate) scales. The adjectives are combined into eight 8item octant scales. Table 1 shows an illustrative adjective from each octant. Some respondents may be unfamiliar with certain adjectives (e.g., "uncrafty"), so a glossary can be provided (Adams \& Tracey, 2004).

IPC measures have often been used to study "interpersonal complementarity" (Sadler, Woody, \& Ethier, this volume). A complementary response to another's behavior is a response that is similar in communion but differs in agency. Some people are less likely than others to make complementary responses, perhaps because they rigidly rely on a narrow range of interpersonal behaviors. One potential indicator of rigidity is the distance the vector sum of an individual's eight octant scores extends from the origin of the IPC. Vector length indexes rigidity because it is greater to the extent that scores are especially high in one segment of the IPC and especially low in the opposite segment. Thus, the longer the vector, the more that individual expresses behaviors exclusively and intensively from that particular segment of the IPC. To test this hypothesis, Tracey (2005, Study 2) had observers watch two participants working together (to concoct a story about an ambiguous picture) and locate the interpersonal meaning of each partner's behavior on the IPC. The results confirmed that participants with longer IAS vectors were less likely to behave in ways that were complementary to their partner's behavior.

The IAS also can help clarify the interpersonal meaning of behaviors or dispositions whose meaning may not be obvious. For example, in order to explore the interpersonal meaning of sexual promiscuity, Markey and Markey (2007) had young men and women complete the IAS and also indicate with how many people they had engaged in various sexual activities. Warm, cold, and dominant individuals reported having more partners for each type of sexual activity than did submissive individuals. Cold and warm individuals reported similar numbers of partners, but presumably cold individuals were driven more by self-focused motives (such as pleasure or narcissistic self-enhancement) and warm individuals were driven more by otherfocused motives (such as closeness and intimacy). 


\section{Assessing Interpersonal Problems}

The eight 8-item scales of the Inventory of Interpersonal Problems (IIP; Horowitz, Alden, Wiggins, \& Pincus, 2000) assess problematic dispositions associated with each octant of the interpersonal circumplex. Table 1 shows illustrative items. Respondents indicate how distressed they have been by each problem on 0 (not at all) to 4 (extremely) scales. The items are divided into two sections: "things you find hard to do with other people" and "things that you do too much".

A number of studies of psychotherapy process and outcome have employed the IIP (Ruiz et al., 2004). Another common use of the IIP has been to identity the interpersonal problems associated with various forms of psychopathology. Whereas some disorders (including most "Axis II" personality disorders) show distinct and consistent IIP profiles, others (including most "Axis I" disorders) do not. For example, Salzer et al. (2008) conducted a cluster analysis on the IIP scores of individuals with generalized anxiety disorder (i.e., chronic, excessive, subjectively uncontrollable worrying about multiple everyday concerns). Four clusters—cold, submissive, intrusive, and exploitable—were identified, suggesting that generalized anxiety may be associated with multiple distinct patterns of interpersonal problems.

The IIP can help guide therapeutic interventions for interpersonal problems. For example, Locke (2005) showed that the interpersonal problems assessed by the IIP are linked to the types of beliefs or interpersonal expectations that are readily targeted by therapeutic interventions. To assess everyday interpersonal expectations, over a one-week period, every time participants imagined how another person might react to them, they wrote down how they expected the other person to react and how that reaction would make them feel. Locke found clear associations between these interpersonal expectations and the interpersonal problems assessed by the IIP. For example, expecting others to be uninviting or unsupportive predicted problems with being too uncommunal; expecting others to be critical (and expecting oneself in 
response to feel angry) predicted problems with being too agentic; and expecting others to be dismissive (and expecting oneself in response to feel ashamed) predicted problems with being too communal. Reducing the frequency or intensity of these negative interpersonal expectations may help people to overcome their chronic interpersonal problems.

\section{Assessing Interpersonal Values and Motives}

Interpersonal values or motives also shape individuals' reactions to interpersonal experiences. For example, being told what to do may be a relief to someone who values submission, but a humiliation to someone who values dominance. Consequently, many psychotherapies try to change feelings and behavior by changing values; for example, cognitive and rational-emotive therapists may help clients question the extreme value they place on certain interpersonal experiences, such as needing approval. The eight 8-item scales of the Circumplex Scales of Interpersonal Values (CSIV; Locke, 2000) assess the value individuals place on interpersonal experiences associated with each octant of the IPC. For each item, respondents indicate how important that type of interpersonal experience is for them on 0 (not important) to 4 (extremely important) scales. Table 1 shows illustrative items.

Interpersonal values can magnify or dampen emotional reactions to interpersonal events.

One common interpersonal event is comparing the self with another person (i.e., a social comparison). Locke (2003) tested if interpersonal values moderate reactions to social comparisons. To assess social comparisons, participants kept a diary of comparisons they made during their everyday lives. For each comparison, they noted (a) whether the other person was better-off, worse-off, similar, or different, and (b) how the comparison made them feel. People with stronger communal values (as assessed by the CSIV) reported stronger positive reactions to noticing that someone was similar, but they did not report stronger-and on some measures reported weaker-reactions to noticing that someone was better-off or worse-off. Thus, interpersonal values moderated the emotional impact of social comparisons. 
Locke and Christensen (2007) found that stronger communal values (as assessed by the CSIV) also predict describing oneself and others in similar terms. (Locke [2009] has since replicated this finding both in the United States and in Korea.) Self-other similarity also correlated negatively with the Machiavellianism scale (MACH; Christie \& Geis, 1970), which measures a detached and manipulative attitude towards others, and positively with the Relational-Interdependent Self-Construal scale (RISC; Cross, Bacon, \& Morris, 2000), which measures tendencies to define oneself in terms of one's close relationships. Because MACH correlates negatively and RISC correlates positively with communal traits and values, inventing separate explanations for the effects of each specific measure was unnecessary; instead, the dimension of communion could explain all three effects. This singular explanation is (a) more parsimonious than having different models for different measures and (b) more generative because it suggests that any interpersonal quality associated with communal motives should predict describing the self and others in similar terms. The broader message is that many measures exist—such as MACH and RISC—-that are designed to assess specific interpersonal dispositions that may not be fully captured by the two-dimensional IPC. Yet, these measures typically correlate with the IPC dimensions (Wiggins \& Broughton, 1991), and locating these measures within the IPC may help us interpret them more effectively. In this way, by using the IPC as a integrative model, we can avoid repeatedly "reinventing the [interpersonal] wheel". Assessing Interpersonal Self-Efficacy

Self-efficacy is a person's confidence that he or she can successfully perform a specific type of action (Bandura, 1997). The eight 4-item scales of the Circumplex Scales of Interpersonal Efficacy (CSIE; Locke \& Sadler, 2007) assess a person's confidence that he or she can successfully perform behaviors associated with each octant of the IPC. For each item, respondents indicate on 0 (not at all confident) to 10 (absolutely confident) scales how sure they are that they could act that way with other people. Table 1 lists illustrative items. 
Locke and Sadler (2007) had pairs of strangers complete the CSIE and (30 minutes later) work together to solve a murder mystery. Greater self-efficacy for enacting dominant than yielding behavior predicted the expression of more dominant behavior during the interaction (as indicated by amount of time spent talking and observers' ratings of dominance). Moreover, pairs who were more similar in self-efficacy for communal actions were more satisfied with their interaction, suggesting that people who are more confident they can be tough than nice and people who are more confident they can be nice than tough may be unlikely to negotiate satisfying working relationships. Collectively, studies using the CSIE and CSIV (plus the study linking the IIP to interpersonal expectations) show the utility of joining the person variables of social-cognitive theory (such as subjective values and self-efficacy) with the IPC model of interpersonal theory.

\section{Observer Ratings of Interpersonal Behavior}

The Chart of Interpersonal Reactions in Closed Living Environments (CIRCLE;

Blackburn \& Renwick, 1996) is a 49-item observer rating scale designed to assess the interpersonal behavior of psychiatric inpatients. Examples of items are "dominates conversations" (PA) and "sits alone or keeps to himself" (FG). The frequency of each behavior is rated on a 4-point scale. The CIRCLE may be particularly useful for inpatient or forensic populations or when self-reports are likely to be invalid. One area of application has been to predict future aggressive behavior in high-risk populations. For example, Doyle and Dolan (2006) had nurses complete the CIRCLE on forensic inpatients. Staff (who had not completed the CIRCLE) then monitored patients' aggressive behavior during the next 12 weeks. Higher ratings on the dominant, coercive, and hostile $(\mathrm{PA}, \mathrm{BC}, \mathrm{DE})$ scales and lower ratings on the compliant (JK) scale predicted future aggression.

The Check List of Interpersonal Transactions (CLOIT; Kiesler, Goldston, \& Schmidt, 1991 ) is a 96-item measure of interpersonal behaviors from each of 16 segments of the IPC. Examples of items are "act in a relaxed, informal, warm, or nonjudgmental manner" (LM) and 
Locke - 10

"act in a stiff, formal, unfeeling, or evaluative manner" (DE). For each item the rater indicates whether or not the target enacted that behavior. The Check List of Psychotherapy Transactions (CLOPT) is a version specifically for ratings of clients or counselors. The CLOIT and CLOPT are not measures of enduring dispositions, and so are most appropriate for identifying patterns of behavior within particular situations or interactions, such as within a therapy session. In recent years researchers have rarely used the entire CLOIT or CLOPT, and instead have been selecting and modifying items from these scales to create their own observational measures. For example, Schmid Mast and Hall (2004) had coders use an aggregate of the CLOIT's dominant and submissive items to rate the dominance of members of male or female dyads engaged in brief interactions. Different coders counted how many times each participant smiled. Perceived dominance was negatively related to smiling among female participants, but positively related to smiling among male participants, suggesting that perceivers may be biased to perceive a woman's smile as deference and a man's smile as confidence.

Assessing Interpersonal Impacts

The eight 7-item octant scales of the Impact Message Inventory-Circumplex (IMI; Kiesler \& Schmidt, 2006; Kiesler, Schmidt, \& Wagner, 1997) assess the interpersonal dispositions of a target person, not by asking the target person directly, but by assessing the "impact messages" (feelings, thoughts, and action tendencies) that the target typically evokes in the respondent. Thus, dominant (PA) scale items assess the reactions a dominant target is likely to evoke such as "[makes me feel] bossed around", whereas submissive (HI) scale items assess the reactions a submissive target is likely to evoke such as "[makes me feel] in charge". Respondents indicate how well each item describes their reaction to the target on not at all (1) to very much so (4) scales. Although the octant scales show a circular ordering around the interpersonal axes, they also show unequal spacing and inconsistent vector 
lengths and so do not meet circumplex criteria as well as the other measures reviewed here (Hafkenscheid \& Rouckhout, 2009; Schmidt, Wagner, \& Kiesler, 1999).

The IMI is generally used to assess the impacts of a specific individual, but it can also be used to assess the impacts of an entire social environment. Gallo, Smith, and Cox (2006) asked participants from community settings to describe their typical social experiences at home, with work supervisors, with coworkers, and with people in their neighborhood on a brief version of the IMI. Having less educational attainment predicted experiencing these social environments as more agentic and uncommunal (i.e., more hostile and controlling). The perception of the social context, particularly the perception of communion, partially mediated the negative association between educational attainment and various self-reported health outcomes. In sum, having less socioeconomic status may result in experiencing less supportive social environments which, in turn, may negatively impact health.

\section{Assessing Social Support Behaviors}

The eight 8-item octant scales of the Support Actions Scale-Circumplex (SAS-C; Trobst, 2000) measure dispositions to provide agentic or communal support to those in need of assistance. Example of items are "give advice" (PA) and "give them a hug" (LM). The SAS-C might be particularly useful for describing the actions of people who are members of support groups or who are providing support to individuals with illnesses or disabilities. Hamann et al. (2008) asked adult siblings to use the SAS-C to describe their supportive behaviors when their brother or sister had a health problem. Specifically, they compared pairs of siblings in which (a) both tested positive for a mutation in the BRCA1/2 gene (which increases cancer risk), (b) both tested negative, or (c) one tested positive and the other tested negative. Siblings with the same test results reported more friendly support behaviors than siblings with different test results, suggesting that having different test results may cause siblings to experience more tension and distance. Also, members of "both positive" dyads reported more dominant support 
behaviors than did members of "both negative" dyads, suggesting that positive dyads may get more actively involved in each other's health needs.

\section{Circles for Children}

Although most research involving the IPC has involved measures developed on and for adults, recently there have been efforts to create IPC inventories specifically for children and adolescents. For example, Sodano and Tracey (2006) created the Child and Adolescent Interpersonal Survey (CAIS), which consists of interpersonal trait descriptions accessible to children such as "I am fun to be around" (NO) and "I call people names" (BC). As another example, Ojanen, Gronroos, and Salmivalli (2005) modified the CSIV to create an "Interpersonal Goals Inventory for Children"; they tried to make the inventory more accessible to children by removing some items and altering others (e.g., changing "not make a social blunder" to "not do anything ridiculous").

\section{Scoring and Interpreting IPC Inventories}

Having chosen and administered an IPC inventory, the next step is to score and interpret the responses. The following section describes simple analytic procedures that can be done without a computer. (For more sophisticated procedures that more fully exploit the inventories' circumplex properties, see Gurtman, this volume.) I will illustrate the procedures using CSIE data, but these same procedures can be used on data from any IPC inventory.

First, compute the raw scale score for each octant. On most IPC inventories, the raw scale scores are positively correlated with each other; this is referred to as the general factor. The general factor may have a substantive meaning or may reflect response tendencies unrelated to item content; for example, both general interpersonal confidence and an acquiescent response style may contribute to the CSIE's general factor. Regardless, for any IPC inventory, the general factor and the individual octant scores have different meanings and so must be examined separately. To accomplish this, separately for each individual, (1) compute the general factor score by averaging the individual's eight octant scores, and then 
(2) "ipsatize" the octant scores by subtracting that individual's general factor score from each raw octant score.

To illustrate, I will analyze the CSIE responses from two participants from Locke and Sadler's (2007) study in which pairs of strangers completed the CSIE before working together to solve a murder mystery. Specifically, I will examine an extremely dissatisfied pair (whose satisfaction with their interaction was 2.4 standard deviations below average). First I computed the raw scale scores and overall mean (or general factor score) for each partner. (Recall that the scores could range from 0 to 10). The overall mean was 7.0 for Partner $A$ and 8.2 for Partner B. Then I ipsatized the scores by subtracting the overall mean from each scale score. Figure 2 plots the ipsatized octant scores for each partner (on scales ranging from a low value of -2.5 at the center of the circle to a high value of 1.5 at the edge of the circle). The figure shows that the two partners were similar in efficacy for being agentic/unagentic, but differed greatly in efficacy for being communal/uncommunal: Partner A was more confident that he could be communal than uncommunal, whereas Partner B was more confident that he could be uncommunal than communal.

The next step is to summarize the individual's overall agentic, unagentic, communal, and uncommunal dispositions by combining the ipsatized octant scores as follows:

$$
\begin{aligned}
& \text { Agentic Vector }=(0.414)(\mathrm{PA}+(0.707)(\mathrm{BC}+\mathrm{NO})) \\
& \text { Unagentic Vector }=(0.414)(\mathrm{HI}+(0.707)(\mathrm{FG}+\mathrm{JK})) \\
& \text { Communal Vector }=(0.414)(\mathrm{LM}+(0.707)(\mathrm{JK}+\mathrm{NO})) \\
& \text { Uncommunal Vector }=(0.414)(\mathrm{DE}+(0.707)(\mathrm{BC}+\mathrm{FG}))
\end{aligned}
$$

The agentic vector minus the unagentic vector yields the individual's overall tendency to be agentic versus unagentic (or vertical or "Y" coordinate). The communal vector minus the uncommunal vector yields the individual's overall tendency to be communal versus uncommunal (or horizontal or " $X$ " coordinate). These $X$ and $Y$ coordinates define a vector sum in the IPC space. The angle of this vector shows the individual's predominant interpersonal 
tendency. The vector length shows how intensely and consistently the target manifests this interpersonal disposition; the longer the vector, the more the scores define a clear interpersonal pattern with a clear peak in one region and a clear trough in the opposite region. For example, returning to our dissatisfied dyad, Partner B's vector angle was 216 degrees (in the FG octant) whereas Partner A's vector angle was 337 degrees (in the JK octant) and his vector length was several times greater than B's (indicating a more perspicuous interpersonal pattern). This mismatch in communal self-efficacy—which may have contributed to a corresponding mismatch in communal behaviors_may be partly to blame for their dissatisfaction.

Because all IPC inventories share the same structure, the same procedures just used to analyze data from the CSIE can be used to analyze data from any IPC inventory. Note also that the procedures just used to analyze an individual's scores also can be used to analyze the scores of a group of individuals-for example, the typical interpersonal style of depressed patients.

\section{Using IPC Measures to Assess Maladaptive Interpersonal Styles}

The IPC model does not define any particular segment of the interpersonal space as necessarily adaptive or maladaptive (Leary, 1957). Indeed, the wide variations in agency and communion across persons and within persons across situations may exist precisely because different levels of agency and communion have both costs and benefits. For example, communion creates opportunities not only for beneficial resource exchange and social support, but also for contracting costly social obligations and diseases. Likewise, agency can increase not only access to valued resources, but also the likelihood of costly rivalries.

Nonetheless, although only the IIP assesses interpersonal problems directly, all IPC measures can help identify maladaptive interpersonal styles. The interpersonal tradition assumes that an adaptive interpersonal style is flexible-that is, able (if necessary) to embody interpersonal behaviors and experiences from any IPC region (Kiesler, 1996; Leary, 1957). In 
contrast, a maladaptive style is too narrow or extreme to be appropriate in many situations. As discussed earlier, one indicator of overly narrow and extreme interpersonal traits is the length of the vector sum of an individual's octant scores (Tracey, 2005).

In addition, conflicted interpersonal profiles (high scores on opposing vectors) may indicate internal ambivalence and a tendency to convey unclear or inconsistent messages (Kiesler, 1996). For example, a person who strongly values both closeness and distance (i.e., who wishes to be loved and embraced but fears being exploited or constrained) may experience distressing internal conflicts and send shifting and confusing messages to others regarding what they want from their relationships.

Finally, particular patterns of scores may be associated with specific psychological disorders, especially personality disorders (PDs) (Horowitz, 2004; Locke, 2006). For example, antisocial and paranoid PDs are associated with "high agency, low communion" interpersonal dispositions; avoidant and schizoid PDs are associated with "low agency, low communion" interpersonal dispositions; dependent PD is associated with "low agency, high communion" interpersonal dispositions; histrionic PD is associated with "high agency, high communion" interpersonal dispositions; and narcissistic PD is associated with high agency interpersonal dispositions.

\section{Conclusions}

IPC inventories offer a balance of comprehensiveness and simplicity: They fully and evenly sample the domain of interpersonal dispositions defined by agency and communion, but enable that information to be distilled into just a few numbers or graphed as a single point on the IPC. In this chapter I showed how the various IPC inventories are being successfully used to investigate a diversity of topics, including interpersonal complementarity, maladaptive expectations, psychopathology, social comparisons, sexuality, relationship satisfaction, and even how socioeconomic factors influence health outcomes. IPC inventories are also being employed in a variety of clinical and therapeutic settings. 
The successful use of the diverse IPC inventories in diverse settings show how the IPC provides a solid two-dimensional foundation—grounded in both theory and research—on which to build a multidimensional understanding of our interpersonal world. Cumulative scientific progress depends on using a consistent set of constructs and locating specific constructs within a more encompassing conceptual framework. By providing a simple yet powerful framework for organizing interpersonal constructs and measures, the IPC is expediting this type of cumulative progress and broad understanding.

If a healthy family is characterized by both differentiation and integration, then the family of IPC inventories I have reviewed in this chapter-while imperfect and open for improvementdoes appear healthy. The sundry inventories are differentiated by their focus on distinct constructs, such as traits, problems, values, self-efficacy, supportive actions, and impacts on others. At the same time, the inventories are anchored in and integrated by the interpersonal circumplex, which remains the most popular and robust model of the cardinal vectors upon which people map and navigate their interpersonal lives. 


\section{References}

Adams, R. S., \& Tracey, T. J. G. (2004). Three versions of the Interpersonal Adjective Scales and their fit to the circumplex model. Assessment, 11, 263-270.

Archer, J. (2006). Testosterone and human aggression: an evaluation of the challenge hypothesis, Neuroscience and Biobehavioral Reviews, 30, 319-345.

Bandura, A. (1997). Self-efficacy: The exercise of control. New York: Freeman.

Bartz, J. A., \& Hollander, E. (2006). The neuroscience of affiliation: Forging links between basic and clinical research on neuropeptides and social behavior. Hormones and Behavior, 50, 518-528.

Blackburn, R., \& Renwick, S. J. (1996). Rating scales for measuring the interpersonal circle in forensic psychiatric patients. Psychological Assessment, 8, 76-84.

Bugental, D. B. (2000). Acquisition of the algorithms of social life: A domain-based approach. Psychological Bulletin, 26, 187-209.

Christie, R. \& Geis, F. (1970). Studies in Machiavellianism. San Diego, CA: Academic Press.

Clark, T. L., \& Taulbee, E. S. (1981). A comprehensive and indexed bibliography of the Interpersonal Check List. Journal of Personality Assessment, 45, 505-525.

Cross, S. E., Bacon, P. L., \& Morris, M. L. (2000). The relational-interdependent self-construal and relationships. Journal of Personality and Social Psychology, 78, 791-808.

Doyle, M. \& Dolan, M. (2006) Evaluating the validity of anger regulation problems, interpersonal style, and disturbed mental state for predicting inpatient violence. Behavioral, Sciences and the Law, 24, 783-798.

Foa, U.G. (1961). Convergences in the analysis of the structure of interpersonal behavior. Psychological Review, 68, 341-353.

Gallo, L.C., Smith, T.W., \& Cox, C. (2006). Socioeconomic Status, Psychosocial Processes, and Perceived Health: An Interpersonal Perspective. Annals of Behavioral Medicine, 31, $109-119$ 
Gurtman, M. B. (2011). Circumplex measures and circular reasoning. In current volume.

Hafkenscheid, A., \& Rouckhout, D. (2009). Circumplex structure of the Impact Message Inventory (IMI-C): an empirical test with the dutch version. Journal of Personality Assessment, 91, 187-94.

Hamann, H., Smith, T. W., Smith, K. R., Ruiz, J. M., Kircher, J. C., Botkin, J.R. (2008). Interpersonal responses among sibling dyads tested for BRCA1/2 gene mutations. Health Psychology, 27, 100-109.

Horowitz, L. M. (2004). Interpersonal foundations of psychopathology. Washington, DC: American Psychological Association.

Horowitz, L. M., Alden, L. E., Wiggins, J. S., \& Pincus, A. L. (2000). Inventory of Interpersonal Problems manual. Odessa, FL: The Psychological Corporation.

Kiesler, D. J. (1996). Contemporary interpersonal theory and research: Personality, psychopathology and psychotherapy. New York: Wiley.

Kiesler, D. J., Goldston, C. S., \& Schmidt, J. A. (1991). Manual for the Check List of Interpersonal Transactions-Revised (CLOIT-R) and the Check List of Psychotherapy Transactions-Revised (CLOPT-R). Richmond: Virginia Commonwealth University.

Kiesler, D. J., \& Schmidt, J. A. (2006). Manual for the Impact Message Inventory-Circumplex (IMI-C). Menlo Park, CA: Mind Garden.

Kiesler, D. J., Schmidt, J. A. \& Wagner, C. C. (1997). A circumplex inventory of impact messages: An operational bridge between emotional and interpersonal behavior. In R. Plutchik, \& H. R. Conte (Eds.), Circumplex models of personality and emotions (pp. 221244). Washington, DC: American Psychological Association.

LaForge, R., \& Suczek, R. F. (1955). The interpersonal dimension of personality: An interpersonal check list. Journal of Personality, 24, 94-112.

Leary, T. (1957). Interpersonal diagnosis of personality. New York: Ronald Press. 
Locke, K. D. (2000). Circumplex Scales of Interpersonal Values: Reliability, validity, and applicability to interpersonal problems and personality disorders. Journal of Personality Assessment, 75, 249-267.

Locke, K. D. (2003). Status and solidarity in social comparison: Agentic and communal values and vertical and horizontal directions. Journal of Personality and Social Psychology, 84, 619-631.

Locke, K. D. (2005). Interpersonal problems and interpersonal expectations in everyday life. Journal of Social and Clinical Psychology, 24, 915-931.

Locke, K. D. (2006). Interpersonal circumplex measures. In S. Strack (Ed.), Differentiating normal and abnormal personality ( $2^{\text {nd }}$ Ed., pp. 383-400). New York: Springer.

Locke, K. D., \& Christensen, L. (2007). Re-Construing the relational self-construal and its relationship with self-consistency. Journal of Research in Personality, 41, 389-402.

Locke, K. D. (2009). Communal values and perceived self-other similarity. Poster session presented at the annual meeting of the Society for Interpersonal Theory and Research, Toronto, Canada.

Locke, K. D., \& Sadler, P. (2007). Self-efficacy, values, and complementarity in dyadic interactions: Integrating interpersonal and social-cognitive theory. Personality and Social Psychology Bulletin, 33, 94-109.

Markey, P. M., \& Markey, C. N. (2007). The interpersonal meaning of sexual promiscuity. Journal of Research in Personality, 41, 1199-1212.

Moskowitz, D. S. (2009). Coming full circle: Conceptualizing the study of interpersonal behaviour. Canadian Psychology, 50, 33-41.

McCrae, R. R., \& Costa, P. T., Jr. (1989). The structure of interpersonal traits: Wiggins' circumplex and the five-factor model. Journal of Personality and Social Psychology, 56, $586-595$. 
Ojanen,T., Gronroos, M., \& Salmivalli, C. (2005) Applying the interpersonal circumplex model to children's social goals: Connections with peer reported behavior and sociometric status. Developmental Psychology, 41, 699-710.

Ruiz, M., Pincus, A., Borkovec, T., Echemendia, R., Castonguay, L., \& Raguesa, S. (2004). Validity of the Inventory of Interpersonal Problems for predicting treatment outcome: An investigation with the Pennsylvania Practice Research Network. Journal of Personality Assessment, 83, 213-222.

Sadler, P., Woody, E., \& Ethier, N. (2011). Complementarity in interpersonal relationships. In current volume.

Salzer, S., Pincus, A. L., Hoyer, J., Kreische, R., Leichsenring, F., \& Leibing, E. (2008). Interpersonal subtypes within generalized anxiety disorder. Journal of Personality Assessment, 90, 292-299.

Schmid Mast, M., \& Hall, J. A. (2004). When is dominance related to smiling? Assigned dominance, dominance preference, trait dominance, and gender as moderators. Sex Roles, 50, 387-399.

Schmidt, J. A., Wagner, C. C., \& Kiesler, D. J. (1999). Psychometric and Circumplex Properties of the Octant Scale Impact Message Inventory (IMI-C): A structural evaluation. Journal of Counseling Psychology, 46, 325-334.

Sodano, S. M. \& Tracey, T. J. G. (2006). Interpersonal traits in childhood and adolescence: Development of the Child and Adolescent Interpersonal Survey. Journal of Personality Assessment, 87, 317-329.

Tracey, T. J. G. (2005). Interpersonal rigidity and complementarity. Journal of Research in Personality, 39, 592-614.

Trobst, K. K. (2000). An interpersonal conceptualization and quantification of social support transactions. Personality and Social Psychology Bulletin, 26, 971-986. 
Wiggins, J. S. (1979). A psychological taxonomy of trait-descriptive terms: The interpersonal domain. Journal of Personality and Social Psychology, 33, 409-420.

Wiggins, J. S. (1995). Interpersonal Adjective Scales: Professional manual. Odessa, FL: Psychological Assessment Resources.

Wiggins, J. S. (2003). Paradigms of personality assessment. New York, NY: Guilford Press.

Wiggins, J. S., \& Broughton, R. (1991). A geometric taxonomy of personality scales. European Journal of Personality, 5, 343-365.

Wiggins, J. S., Trapnell, P., \& Phillips, N. (1988). Psychometric and geometric characteristics of the Revised Interpersonal Adjective Scales (IAS-R). Multivariate Behavioral Research, 23, 517-530. 
Table 1

Examples of Items From Each Octant of the IAS, IIP, CSIV, and CSIE

\begin{tabular}{|c|c|c|c|c|}
\hline Octant Scale & $\begin{array}{l}\text { Example IAS Items } \\
\text { (Rate how accurately } \\
\text { each describes you) }\end{array}$ & \begin{tabular}{|l} 
Example IIP Items \\
(Rate how distressing each \\
problem has been)
\end{tabular} & $\begin{array}{l}\text { Example CSIV Items } \\
\text { "When I am with him/her/them, } \\
\text { it is important that..." }\end{array}$ & $\begin{array}{l}\text { Example CSIE Items } \\
\text { "Rate how confident you are that } \\
\text { you can..." }\end{array}$ \\
\hline $\begin{array}{l}\text { LM } \\
\text { (communal) }\end{array}$ & Sympathetic & $\begin{array}{l}\text { I try to please other people too } \\
\text { much }\end{array}$ & ... I feel connected to them & ...be helpful \\
\hline $\begin{array}{l}\text { NO } \\
\text { (agentic \& communal) }\end{array}$ & Perky & $\begin{array}{l}\text { I tell personal things to other } \\
\text { people too much }\end{array}$ & ...they respect what I have to say & ...express myself openly \\
\hline $\begin{array}{l}\text { PA } \\
\text { (agentic) }\end{array}$ & Forceful & $\begin{array}{l}\text { I try to control other people too } \\
\text { much }\end{array}$ & $\begin{array}{l}\text {...they acknowledge when I am } \\
\text { right }\end{array}$ & ...be assertive \\
\hline $\begin{array}{l}\text { BC } \\
\text { (agentic \& uncommunal) }\end{array}$ & Boastful & $\begin{array}{l}\text { I fight with other people too } \\
\text { much }\end{array}$ & ...I keep the upper hand & ...be aggressive if I need to \\
\hline $\begin{array}{l}\mathrm{DE} \\
\text { (uncommunal) }\end{array}$ & Ruthless & $\begin{array}{l}\text { It is hard for me to show } \\
\text { affection to people }\end{array}$ & $\begin{array}{l}\text {...they keep their distance from } \\
\text { me }\end{array}$ & ...get them to leave me alone \\
\hline $\begin{array}{l}\text { FG } \\
\text { (unagentic \& uncommunal) }\end{array}$ & Unsociable & I am too afraid of other people & ...I not say something stupid & ...hide my thoughts and feelings \\
\hline $\begin{array}{l}\text { HI } \\
\text { (unagentic) }\end{array}$ & Timid & $\begin{array}{l}\text { It is hard for me to be assertive } \\
\text { with another person }\end{array}$ & ...I not make them angry & ...be a follower \\
\hline $\begin{array}{l}\text { JK } \\
\text { (unagentic \& communal) }\end{array}$ & Unargumentative & I am too gullible & ...they like me & ...get along with them \\
\hline
\end{tabular}


Locke - 23

Figure 1. The interpersonal circumplex

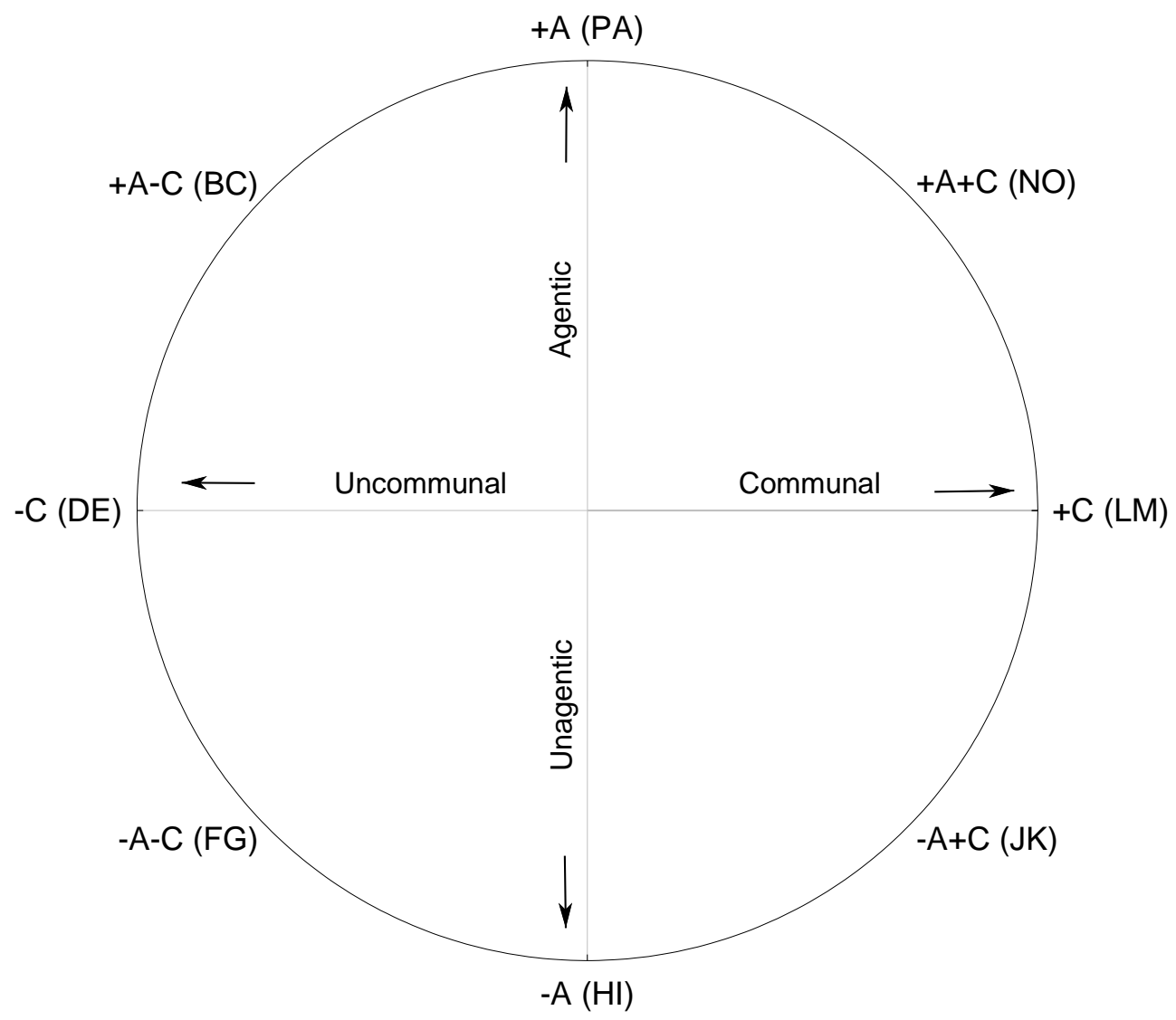


Figure 2. CSIE octant scores of a pair dissatisfied participants from Locke and Sadler (2007. Study 2). Partner A's scores are connected by a dashed line; Partner B's scores are connected by a solid line.

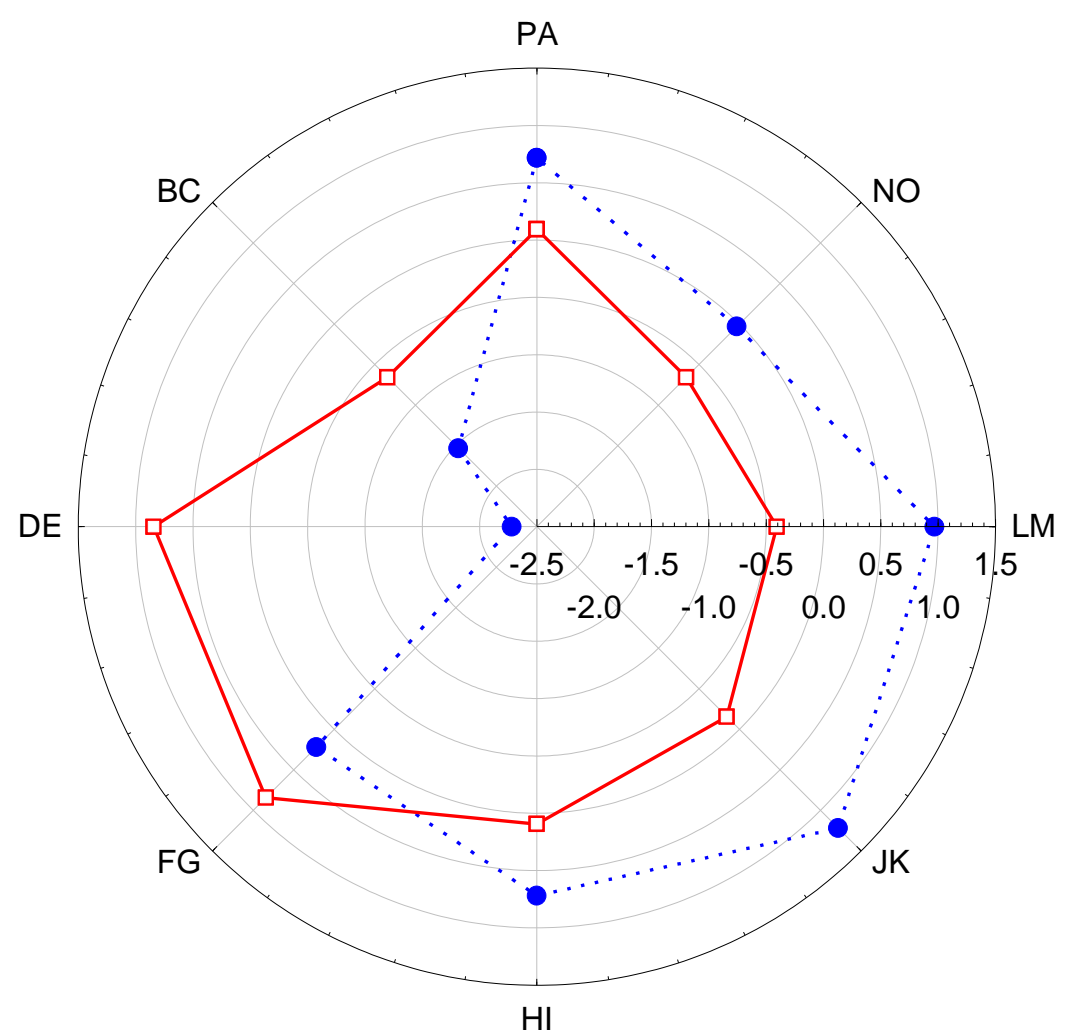

